\title{
Use of Electromagnetic Midsagittal Articulography in the Study of Swallowing
}

Catriona M Steele; Pascal H H M Van Lieshout

Journal of Speech, Language, and Hearing Research; Apr 2004; 47, 2; Wilson Education Abstracts pg. 342

\section{Use of Electromagnetic Midsagittal Articulography in the Study of Swallowing}

RESEARCH NOTE

\author{
Catriona M. Steele \\ Pascal H. H. M. Van Lieshout \\ University of Toronto, \\ Toronto, Ontario, Canada
}

The tongue functions as the primary articulator during the oropharyngeal stages of swallowing. However, detailed descriptions of the kinematics and spatiotemporal variability of tongue behaviors during swallowing are limited to a handful of analyses of data from the $X$-ray microbeam database. In this article, a new technique, electromagnetic midsagittal articulography (EMMA), is introduced for the high-resolution description of oral articulatory movements during swallowing. Data from 8 healthy, nondysphagic participants are used to illustrate the methods used for data collection and analysis. Movement data were collected for 3 fleshpoint positions on the tongue (blade, body, dorsum) during sequences of repeated discrete water swallows, and were characterized for variables of spatiotemporal variability and 4 discrete kinematic parameters (movement amplitude, peak velocity, duration, and kinematic stiffness). These data show that the movement trajectories measured using EMMA are consistent with descriptions from previous $X$-ray microbeam studies, indicating that EMMA is a feasible method for the detailed study of tongue movements during swallowing.

KEY WORDS: electromagnetic midsagittal articulography, swallowing, dysphagia, kinematics, tongue 
exposure. However, the X-ray microbeam technique has limited practical value due to the inaccessibility of the required equipment.

In the past 10-15 years, electromagnetic midsagittal articulography (EMMA) has been used for the parameterization of multiple midsagittal tongue fleshpoint movement during speech production (e.g., Engelke, Schönle, Kring, \& Richter, 1989; Van Lieshout, Alfonso, Hulstijn, \& Peters, 1994). The electromagnetic midsagittal articulograph (Carstens Medizinelektronik AG-100, Lenglern, Germany) traces the position of very small transducer coils (each $2.4 \mathrm{~mm}$ in diameter) that are attached in midline to the oral articulators, as shown in Figure 1. A recent study (Byrd, Browman, Goldstein, \& Honorof, 1999) compared EMMA speech data with $\mathrm{X}$-ray microbeam data and concluded that the results obtained from both systems are highly comparable. Previous authors have suggested that EMMA might have application for the study of intra-oral movements during swallowing (Engelke et al., 1989). To date, however, published reports of such data are limited to abstracts from scientific meetings (Steele \& Van Lieshout, 2002).

We describe the use of EMMA to measure tongue movements during swallowing. Like other methods used to study tongue kinematics (X-ray microbeam, EPG, U/ S), EMMA does not allow concurrent visualization of tongue fleshpoint position and bolus size, shape, or location. Thus, it is not possible to map EMMA data onto the oral preparatory, oral propulsive, and pharyngeal stages of bolus movement. However, EMMA does provide a detailed spatiotemporal assessment of oral tongue

Figure 1. Electromagnetic midsagittal articulography (EMMA) transducer coil positions. During EMMA studies of swallowing, transducer coils are attached in midline to the fleshpoint positions shown in the diagram. Reference coils are denoted by the letter $R$.

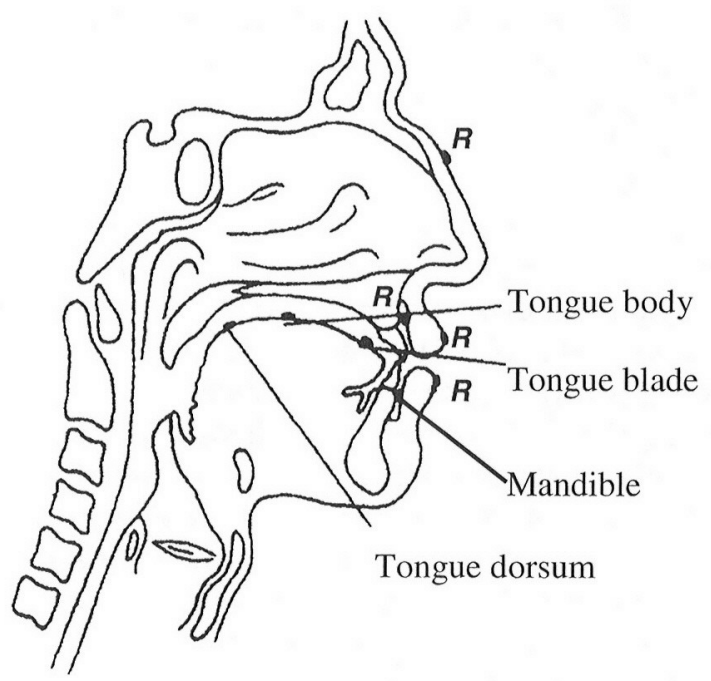

movements as they unfold over the course of the bolus propulsion sequence. EMMA also offers advantages over videofluoroscopic and X-ray microbeam methods, in that it permits both extended and multiple, repeated data collection sessions for individual participants, without risk of significant biohazard (Hasegawa-Johnson, 1998). This facilitates the study of variability in tongue behaviors for swallowing over time (Steele \& Van Lieshout, 2003).

To illustrate the EMMA technique, data from 8 healthy adults are presented in this article. Demographic characteristics of the participants are summarized in Table 1. All participants provided informed consent to participate, completed a brief medical history questionnaire, and were judged to have normal oral motor function during a screening by the first author, a registered speech-language pathologist.

\section{The EMMA Procedure}

In our EMMA swallowing study protocol, three transducer coils are attached in midline to the surface of the tongue using a combination of surgical methacrylate resin (Cyanodent; Ellman International, Inc., Hewlett, NY) and zinc polycarboxylate dental cement (Durelon; 3M/Espe Dental, St. Paul, MN). The most anterior coil (tongue blade) is positioned $10 \mathrm{~mm}$ posterior to the anatomical tongue tip. A middle coil (tongue body) is positioned $20 \mathrm{~mm}$ behind the blade coil and the third coil is positioned on the tongue dorsum (as far back as tolerated by the participant). Exact intercoil distances can then be measured (in millimeters posterior to the tongue blade coil) on the nonextended tongue from the EMMA data, as shown in Table 1. The coil locations recorded for the 8 participants in this article are comparable to the $\mathrm{T} 1, \mathrm{~T} 2$, and $\mathrm{T} 3$ pellet locations used in previous X-ray microbeam studies (Martin, 1991; Tasko et al., 2002). We also attach a fourth coil to the mandibular incisors, using a custom thermoplastic dental impression; this allows for measurement of jaw movement and corrections of the tongue coil data for jaw contributions. Four additional transducer coils are attached in midline to the nose, the vermilion borders of the upper and lower lip, and the gums of the upper central incisors to provide reference data.

Following coil attachment, the participant is seated comfortably in a dental chair, with his or her head positioned inside a large plastic helmet $(62 \mathrm{~cm})$ that is suspended from the ceiling. Three transmitters attached to this helmet create an alternating magnetic field within the helmet. When a transducer coil is placed inside this field, an alternating voltage is induced in the transducer. The distance between the transmitter and the transducer is directly related to the amplitude of the signal 
Table 1. Participant demographics and transducer coil positions.

\begin{tabular}{|c|c|c|c|c|c|c|c|c|c|c|c|c|c|}
\hline \multirow[b]{2}{*}{ Gender } & \multirow[b]{2}{*}{ Participant } & \multirow[b]{2}{*}{ Age } & \multicolumn{2}{|c|}{ Session } & \multirow{2}{*}{$\begin{array}{l}\text { Tongue blade } \\
\text { (mm posterior } \\
\text { to anatomical } \\
\text { tongue tip) }\end{array}$} & \multicolumn{4}{|c|}{$\begin{array}{c}\text { Tongue body } \\
\text { (mm posterior to tongue blade coil) }\end{array}$} & \multicolumn{4}{|c|}{$\begin{array}{c}\text { Tongue dorsum } \\
\text { (mm posterior to tongue blade coil) }\end{array}$} \\
\hline & & & A & B & & Session A & Session B & $M$ & $S D$ & Session A & Session B & $M$ & $S D$ \\
\hline \multirow[t]{5}{*}{ Females } & 2 & 28 & 3 & $4^{a}$ & 10.00 & 17.31 & 21.28 & 19.30 & 2.81 & 36.59 & 39.54 & 38.07 & 2.09 \\
\hline & 4 & 28 & 1 & 2 & 10.00 & 22.46 & 29.72 & 26.09 & 5.13 & 41.72 & 38.39 & 40.06 & 2.35 \\
\hline & 3 & 59 & 2 & 3 & 10.00 & 21.27 & 27.59 & 24.43 & 4.47 & 32.57 & 35.47 & 34.02 & 2.05 \\
\hline & 8 & 56 & 2 & 3 & 10.00 & 21.80 & 20.83 & 21.32 & 0.69 & 31.60 & 38.87 & 35.24 & 5.14 \\
\hline & & & & & & \multicolumn{2}{|c|}{ Group: } & 22.78 & 3.27 & \multicolumn{2}{|c|}{ Group: } & 36.84 & 2.91 \\
\hline \multirow[t]{5}{*}{ Males } & 1 & 27 & 2 & $4^{a}$ & 10.00 & 28.95 & 16.95 & 22.95 & 8.49 & 34.78 & 21.28 & 28.03 & 9.55 \\
\hline & 5 & 23 & 2 & 3 & 10.00 & 21.87 & 26.68 & 24.28 & 3.40 & 36.02 & 36.99 & 36.51 & 0.69 \\
\hline & 6 & 66 & 1 & 3 & 10.00 & 26.11 & 26.22 & 26.17 & 0.08 & 35.65 & 33.49 & 34.57 & 1.53 \\
\hline & 7 & 56 & 1 & 2 & 10.00 & 23.63 & 32.31 & 27.97 & 6.14 & 47.04 & 54.26 & 50.65 & 5.11 \\
\hline & & & & & & \multicolumn{2}{|c|}{ Group: } & 25.35 & 4.53 & \multicolumn{2}{|c|}{ Group: } & 37.44 & 4.22 \\
\hline
\end{tabular}

Note. Coil positions are shown for two data collection sessions, selected for analysis from a series of three or more repeated data collection sessions per participant. The tongue blade coil position was always $10 \mathrm{~mm}$ posterior to the anatomical tongue tip.

a Participants 1 and 2 each attended a total of four data collection sessions. All other participants attended three data collection sessions.

induced in that transducer (Van Lieshout et al., 1994), allowing precise determination of the location of each coil (spatial error is $<0.35 \mathrm{~mm}$ ). A specially designed inner helmet is tightly connected to the participant's head and to the larger outer helmet, so that head movements are closely followed by the entire structure. Small deviations or rotations of the transducer coils away from midline (up to $1 \mathrm{~cm}$ lateral deviation and $30^{\circ}$ rotation) can be corrected using the signal of the third transmitter (Van Lieshout et al., 1994). Stronger deviations can be detected on screen while monitoring the online data collection. Error signals corresponding to misalignment are stored for future use and can be used to determine whether any signals should be discarded from the data set.

Temporal resolution of the EMMA system is limited only by the memory and processing capacity of the computer used to store the data. For the present illustration, coil-position data were acquired at $400 \mathrm{~Hz}$. Timelocked measurements of the acoustics of swallowing (see Figure 2) were also acquired at $16 \mathrm{kHz}$ using a nonmetallic Littman Cardiology II stethoscope head, connected to a small microphone and secured around the neck with a Velcro strap. The stethoscope head was positioned on the left side of the neck, just inferior to the thyroid lamina (Cichero \& Murdoch, 2002). The acoustic data were collected for the purposes of indexing the number of swallows in each EMMA recording window, using the acoustic swallow peaks, which have previously been reported to coincide with bolus passage through the pharyngo-esophageal segment (Hamlet, Nelson, \& Patterson, 1990).

\section{Tasks}

Prior to the collection of swallowing movement data with the EMMA system, we recommend that participants perform a series of speech and nonspeech oral movement tasks; these serve the purpose of providing baseline static and dynamic position measurements, and allow participants to become fully acclimatized to the presence of the transducer coils. In our protocol, baseline tasks include an occlusal plane measurement, which is taken from two transducer coils, mounted with $3 \mathrm{~cm}$ separation on a plastic bite plate. In this research note, for illustrative purposes, we report swallowing data from 256 discrete water swallows, collected over two data collection sessions for each participant. These data were collected in sets (henceforth called trial-sets) of 8 repeated swallows, performed in series (i.e., 32 swallows per participant, collected in four trial-sets per participant, with two trialsets in each of two data collection sessions per participant). Each trial-set was easily completed within a 40 -s EMMA recording window. Discrete swallows (i.e., single, isolated swallow tokens) serve as a useful referent task, because they have been the predominant task paradigm in previous studies of swallowing biomechanics (ChiFishman et al., 1998). In the present study, the participants were instructed to take eight single sips in sequence, lowering the container of liquid slightly from their lips between each sip. No instructions were given to hold the bolus in the mouth prior to initiating the swallow or to initiate bolus transport; thus, the data for each discrete swallow event include natural behaviors associated with bringing the cup to the lips, extracting the bolus, positioning it for transport, and executing the swallow. 
Figure 2. Example of EMMA data for three swallows in a trial-set of discrete water swallows. Data are shown for Participant 4. At the top of the figure, the acoustic signal shows a series of three bursts, each representing bolus flow from the pharynx into the esophagus. Vertical dashed lines descending from each swallow peak divide the vertical movement traces for each transducer coil into segments associated with each swallow. Positional peaks and valleys detected by an automated algorithm based on the cyclic spatiotemporal index are identified by upward and downward facing triangles in each movement signal. The tongue blade leads the movement sequence, displaying two complete cycles of upward and downward movements prior to each acoustic swallow burst. Tongue blade movements are followed by single cycles of upward and downward deflections in the tongue body and tongue dorsum for each swallow. Labeled arrows illustrate the durational and amplitude measurements calculated from the movement data.

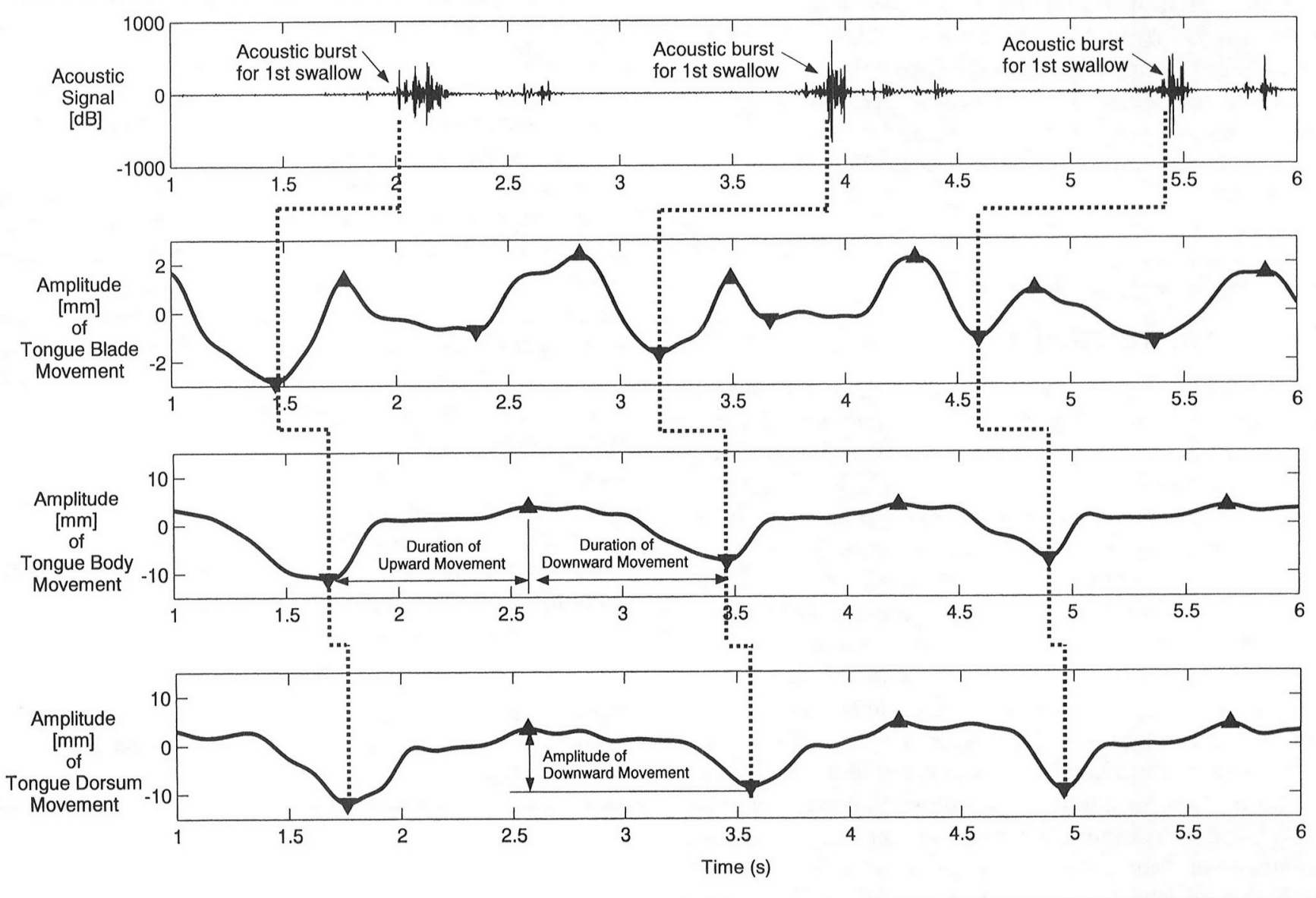

\section{Processing}

The procedures that we use for processing EMMA swallowing data follow the same principles described for previous speech studies (Van Lieshout \& Moussa, 2000). Movement data are first smoothed using an 11point triangular filter (effective low pass frequency = $27.5 \mathrm{~Hz}$ ). Position-reference information from the occlusal plane measurement is then used to rotate the data and align them with the horizontal axis of the EMMA measurement field. In this manner, a uniform coordinate reference frame is established for all participants (Westbury, 1994). Movement data are then imported into MATLAB (Version 6.0.0.42a, Release 12; The Mathworks, Inc., 2001) and band-pass filtered between $0.1 \mathrm{~Hz}$ (removing slow varying drifts) and $6 \mathrm{~Hz}$ using a 7th-order Hamming window Butterworth filter.
Tongue coil signals are corrected for jaw movements using an estimate of jaw rotation, based on the principal component of the mandible transducer coil trajectory for each trial. Compared to a simple subtraction method, which ignores jaw rotation, this method reduces positional and speed errors by approximately $65 \%-70 \%$ (Westbury, 1994).

\section{Event Indexing}

Following processing and filtering, the resulting EMMA data signals consist of oscillatory position and velocity data for each transducer coil. Individual movement cycles can be indexed using either manual procedures or algorithms. In our laboratory, we use an automated peak-picking algorithm to detect peaks and 
valleys in the position and velocity signals (i.e., changes in movement direction; Van Lieshout, Rutjens, \& Spauwen, 2002). The optimum time and amplitude criteria for peak and valley selection for each transducer coil (i.e., those that are used to define the boundaries of the movement segments used for the kinematic analysis) are those characterized by the lowest cyclic spatiotemporal index (cSTI), a measure of spatiotemporal pattern variability, which is defined below (Smith, Goffman, Zelaznik, Ying, \& McGillem, 1995). Use of the cSTI to determine the optimal relative time and amplitude criteria for data segmentation provides an objective method for separating individual cycles in oscillatory oral articulatory movement data (Van Lieshout et al., 2002).

\section{Illustrative Results Movement Trajectories}

An example of EMMA movement data (for Participant 4) is shown in Figure 2. The acoustic waveform is shown at the top of the figure, with position traces for vertical displacement of the tongue blade, body, and dorsum coils below. In anticipation of each acoustic swallow burst, a sequence of upward and downward transducer coil movements is observed, led by the tongue blade. In this example, the tongue blade shows two complete movement cycles prior to each acoustic swallow burst in the series. In the tongue body and dorsum, a single cycle of upward and downward deflections is seen for each swallow-to-swallow interval. A positional plateau at maximum height can be observed in the tongue body and dorsum signals; this is presumed to correspond to full-palate contact. The observed sequence of movements is consistent with the stages previously described in X-ray microbeam studies of swallowing (e.g., Martin, 1991).

Figure 3 illustrates the two-dimensional movement paths of the three tongue transducer coils, over a com. plete trial-set of eight repeated discrete water swallows from the same participant. Consistent with Martin's (1991) X-ray microbeam data, these data show larger and more spatially complex movement trajectories for the tongue body and dorsum coils compared to the tongue blade. In the illustrated example, all three coils show greater vertical displacement than horizontal displacement. Figure 4 presents individual coil trajectories for a single movement cycle, extracted from the same trialset portrayed in Figure 3. The tongue body and tongue dorsum both display a pattern of anterior movement at maximum height during the middle portion of the movement. This observation concurs with previous descriptions of anterior tongue "drifting" during full tonguepalate contact (Martin, 1991).

\section{cSTI}

The cSTI is a measure of spatiotemporal pattern variability (Van Lieshout et al., 2002). To calculate cSTI, the positive position and velocity signals are first amplitude- and time-normalized and aligned with each other, as illustrated in the lower three bars of Figure 5 . Separate standard deviations for the overlapping segments are then computed at $2 \%$ intervals in relative time. The cSTI is defined as the sum of these standard deviations. The cSTI has not been used as a variable in previous studies of swallowing; we propose that comparisons of swallowing cSTI between healthy and clinical participant populations may provide new and interesting insights regarding oral movement variability and its contribution to the integrity of swallowing. Descriptive statistics for cSTI from the present data set, summarized by session, are shown in Table 2 . Repeated-measures analyses of variance (ANOVAs) were performed to identify any main effects of session (Session A or Session B) on CSTI ; none of the comparisons reached an $F$ value higher than 1 , except for tongue blade in the vertical dimension, $F(1,6)=2.75, p=.15$. This finding of stability in the spatiotemporal signature of swallowingrelated tongue behaviors across sessions concurs with X-ray microbeam findings reported by Martin (1991) and suggests that future studies may be able to pool data for equivalent tasks across data collection sessions. However, the reader is reminded that the present analysis

Figure 3. Two-dimensional trajectories of tongue fleshpoint movement during a complete trial-set of eight repeated discrete water swallows.

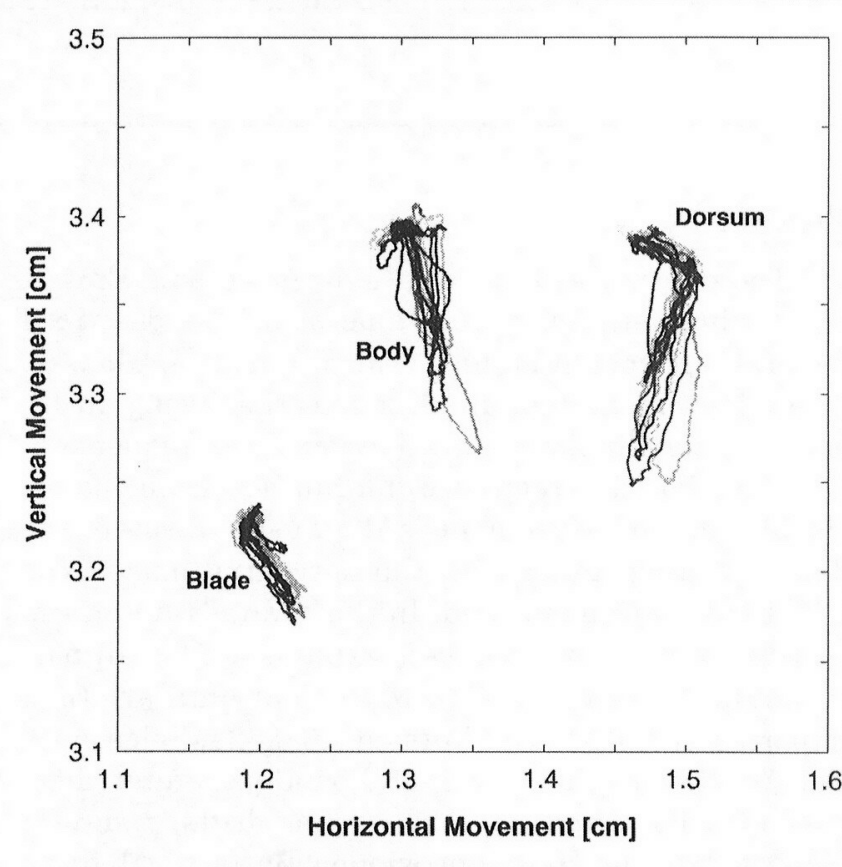


Figure 4. Individual $X-Y$ movement trajectories for the tongue blade, body, and dorsum for a single movement cycle during discrete water swallows.
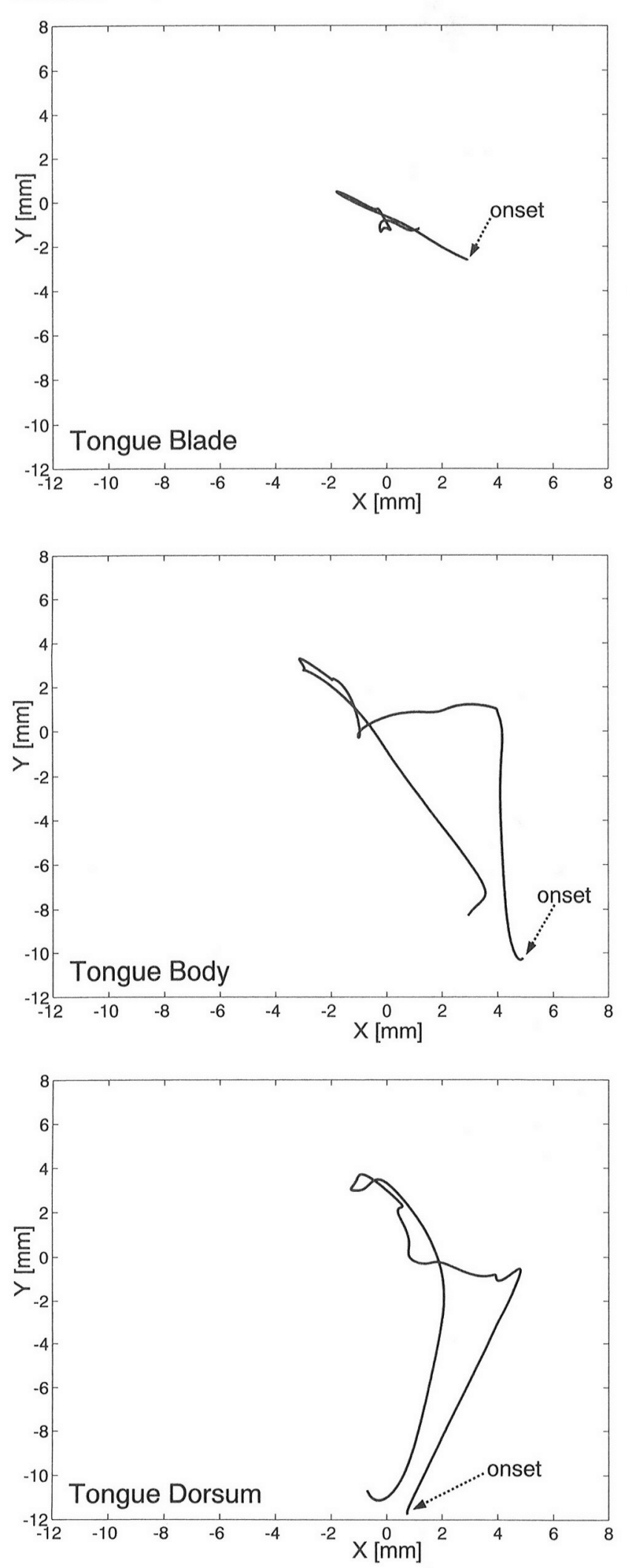

was conducted on a limited number of healthy participants and was restricted to data for a single liquid (water). Thus, the conclusion that swallowing behaviors do not vary markedly across sessions cannot be generalized beyond the strict conditions of the present analysis. It remains to be determined whether greater variability in swallowing behaviors will emerge across an increased selection of bolus textures and flavors. Similarly, it remains unknown whether individuals with dysphagia display similar stability in oral articulatory movements across sessions. These caveats notwithstanding, the present results can also be taken as evidence that the EMMA system measures lingual swallowing behaviors reliably across sessions.

\section{Average Number of Movement Cycles per Swallow}

A single movement cycle in the vertical or horizontal plane would be defined as a complete oscillation (i.e., up + down, or forward + backward), falling between any two successive peaks or valleys in the movement signal (determined using the event indexing procedure described above). It is important to realize that a single movement cycle does not necessarily coincide with a single swallow. Each swallow-to-swallow interval in the EMMA data comprises all bolus events prior to transit through the pharyngo-esophageal segment; in the case of discrete swallows performed in series, these events include positioning of the cup at the lips, and extraction of the liquid from the cup. Multiple movement cycles may be observed for each tongue segment within a single swallow-to-swallow interval (see Figure 2); this fact has not been recognized in previous swallowing literature. The average number of movement cycles per swallow can be calculated as the total number of movement cycles in the trial-set, divided by the number of swallows in the trial-set (eight). We propose that this variable may be particularly useful for studying age-related changes in tongue behaviors for swallowing, given recent reports that elderly participants more commonly display multipeaked tongue gestures during pressure generation for bolus propulsion (Nicosia et al., 2000). Indeed, the present data set illustrates the sensitivity of this variable to age-related differences. Descriptive statistics for the average number of tongue dorsum movement cycles per swallow in the present data set are tabulated by cohort in Table 3. Repeated-measures ANOVAs with a between-subjects factor of cohort (younger or older) and a within-subject factor of session (Session A or Session B) identified significantly more vertical tongue dorsum movement cycles per swallow in the older participants $(M=2.2)$ than in the younger cohort $(M=0.85), F(1,6)$ $=23.83, p=.003$, power $=.781$ (where power is 1 minus the probability of Type II error). 
Figure 5. Detection of multiple oscillations per swallow-to-swallow interval using the cyclic spatiotemporal index (cSTI) algorithm.

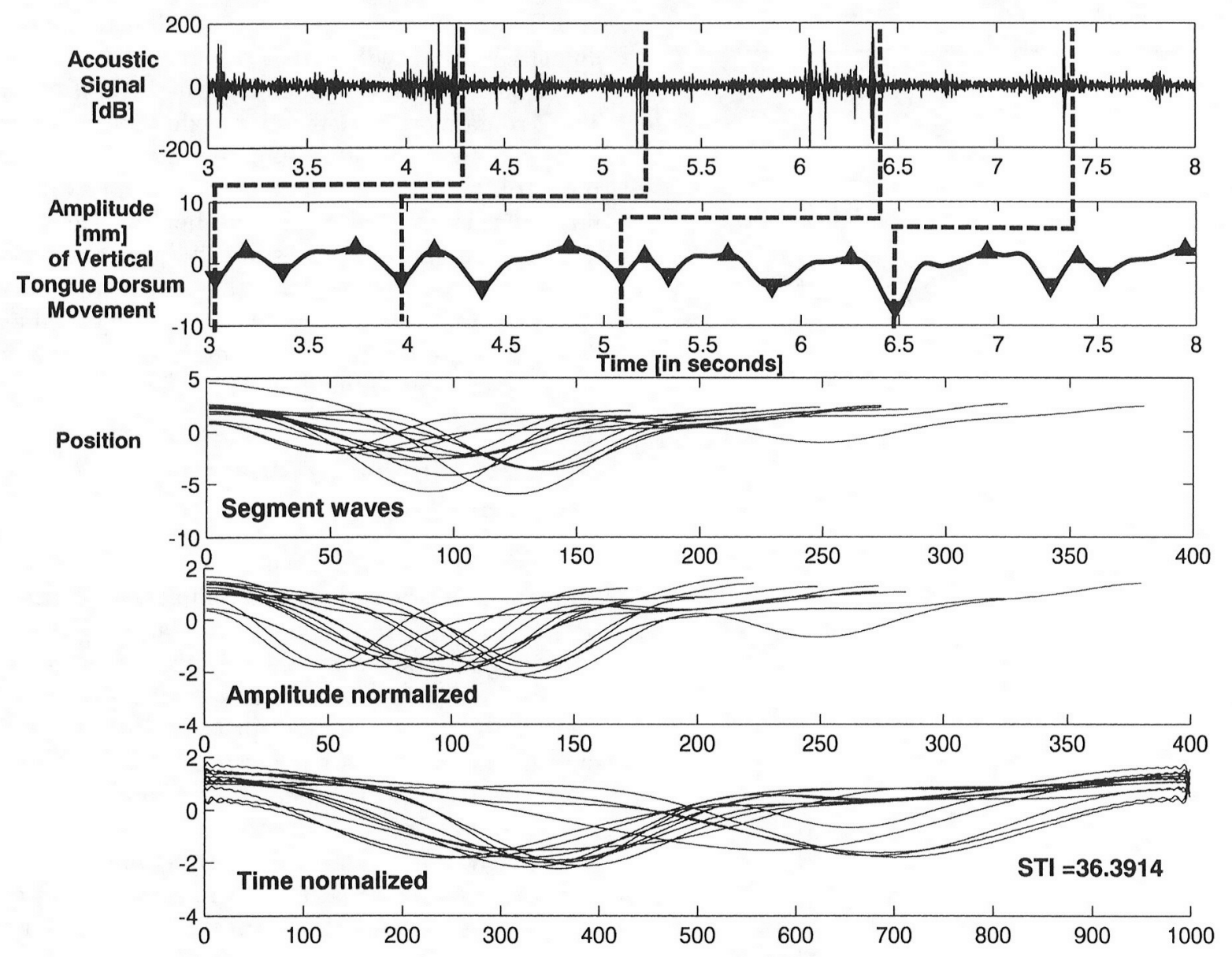

\section{Kinematic Analyses}

Kinematic measurements can be made on EMMA data to characterize individual articulatory movements (i.e., the segment between any two adjacent positional peaks and valleys). For the present illustrative analysis, four kinematic variables were studied: amplitude, peak velocity, duration, and the kinematic stiffness coefficient (defined below). These variables reflect basic characteristics of individual fleshpoint movements and have previously been used in kinematic studies of speech (e.g., Munhall, Ostry, \& Parush, 1985; Perkell \& Zandipour, 2002) and X-ray microbeam swallowing data (Martin, 1991). Using Figure 2 to illustrate these variables, amplitude would be defined as the magnitude of position change between any contiguous pair of peaks and valleys; a single example is shown for downward movement of the tongue dorsum. Peak velocity would represent the maximum velocity (in millimeters per second) measured within each position change. Movement duration would be measured as the difference (in milliseconds) between onset and offset of the position change; Figure 2 shows examples for upward and downward movement of the tongue body. The kinematic stiffness coefficient is a derived measure of mass-normalized kinematic stiffness defined in the context of a mass-spring model (Perkell $\&$ Zandipour, 2002), and reflects the scaling relationship between peak velocity and movement amplitude (stiffness $=$ peak velocity/amplitude).

In the present analysis, four mean trial-set values were calculated for each kinematic variable (one for each movement direction: up, down, forward, and backward) across all the individual movement cycles in the trialset. Additionally, for each kinematic variable, directionspecific standard deviations for each trial-set were calculated as a measure of within-trial-set variation. Previous studies of swallowing kinematics have focused predominantly on the single, discrete swallow; consequently, systematic patterns with respect to variation across repeated swallows within a sequence have not been described. EMMA allows us to collect a large quantity of data from individual participants without risk of biohazard, thereby facilitating the study of variability across a greater number of swallows than is possible with radiographic methods. 
Table 2. Cyclic spatiotemporal index (cSTI): Cohort means and standard deviations for vertical and horizontal tongue movement CSTI (blade, body, and dorsum), measured over series of repeated discrete water swallows.

\begin{tabular}{cccccc}
\hline & \multicolumn{2}{c}{ Vertical cSTI } & & \multicolumn{2}{c}{ Horizontal cSTI } \\
\cline { 2 - 3 } \cline { 5 - 6 } & Session A & Session B & & Session A & Session B \\
\hline N (trial-sets) & 16 & 16 & & 16 & 16 \\
Tongue blade & & & & \\
M & 29.29 & 31.91 & & 30.91 & 31.14 \\
SD & 4.49 & 2.77 & & 4.39 & 3.85 \\
Tongue body & & & & \\
M & 33.02 & 30.80 & & 30.59 & 30.26 \\
SD & 6.27 & 7.08 & & 6.78 & 7.38 \\
Tongue dorsum & & & & & \\
M & 27.43 & 25.80 & & 30.56 & 29.99 \\
SD & 6.00 & 6.64 & & 2.70 & 3.77 \\
\hline
\end{tabular}

aEach trial-set comprises a series of eight repeated discrete water swallows.

Descriptive statistics for the trial-set means and within-trial-set variation (standard deviations) for the four kinematic variables (amplitude, peak velocity, duration, and kinematic stiffness) are tabulated by transducer coil and movement direction in Tables 4 and 5, respectively. Inspection of these data reveals that vertical movement amplitudes in the tongue body and dorsum were larger and showed greater within-trial-set
Table 3. Average number of vertical and horizontal tongue dorsum movement cycles per swallow by cohort for trial-sets of repeated discrete water swallows.

\begin{tabular}{|c|c|c|c|c|c|}
\hline \multirow[b]{2}{*}{ Cohort } & \multirow{2}{*}{$\stackrel{N}{N}$} & \multicolumn{2}{|c|}{$\begin{array}{c}\text { Vertical } \\
\text { movement }\end{array}$} & \multicolumn{2}{|c|}{$\begin{array}{l}\text { Horizontal } \\
\text { movement }\end{array}$} \\
\hline & & $M$ & $S D$ & $M$ & $S D$ \\
\hline Younger $(<30)$ & 16 & 0.9 & 0.5 & 2.4 & 1.3 \\
\hline Older (>50) & 16 & 2.2 & 0.9 & 2.2 & 0.8 \\
\hline
\end{tabular}

aEach trial-set comprises a series of eight repeated discrete water swallows.

variation than corresponding horizontal movement amplitudes for the same tongue segments. Table 4 shows that the highest peak velocities were seen for upward movement in the tongue body. Similarly, in the tongue dorsum, peak velocities for movements in the vertical dimension were higher than those observed in the horizontal plane of movement. Inspection of the trial-set mean data for movement duration (see Table 4) shows an interesting trading relationship between the tongue body and dorsum; namely, in the tongue body, the longest and most variable movement durations were observed in the forward direction, while tongue dorsum movements were of maximal average duration in the upward direction.

The observed mean values for amplitude in the present analysis (see Table 4) are comparable to distance measurements reported by Tasko et al. (2002) for T2 and

Table 4. Descriptive statistics for trial-set mean values of movement amplitude, peak velocity, duration, and kinematic stiffness during discrete water swallows.

\begin{tabular}{|c|c|c|c|c|c|c|c|c|c|}
\hline & \multirow[b]{2}{*}{$N^{a}$} & \multicolumn{2}{|c|}{ Up } & \multicolumn{2}{|c|}{ Down } & \multicolumn{2}{|c|}{ Forward } & \multicolumn{2}{|c|}{ Backward } \\
\hline & & $M$ & $S D$ & $M$ & $S D$ & $M$ & $S D$ & $M$ & $S D$ \\
\hline \multicolumn{10}{|l|}{ Tongue blade } \\
\hline Amplitude (mm) & 32 & 3.07 & 1.56 & 3.04 & 1.56 & 2.97 & 1.10 & 3.00 & 1.12 \\
\hline Peak velocity $(\mathrm{mm} / \mathrm{s})$ & 32 & 17.47 & 9.64 & 18.29 & 10.44 & 16.71 & 7.46 & 17.63 & 8.53 \\
\hline Duration (s) & 32 & 0.53 & 0.19 & 0.56 & 0.22 & 0.60 & 0.26 & 0.57 & 0.27 \\
\hline Kinematic stiffness $\left(\mathrm{s}^{-1}\right)$ & 32 & 6.48 & 1.57 & 7.29 & 2.53 & 6.89 & 1.68 & 6.75 & 1.69 \\
\hline \multicolumn{10}{|l|}{ Tongue body } \\
\hline Amplitude (mm) & 32 & 6.08 & 3.20 & 6.05 & 3.31 & 5.01 & 1.70 & 5.07 & 1.69 \\
\hline Peak velocity $(\mathrm{mm} / \mathrm{s})$ & 32 & 38.49 & 22.42 & 26.13 & 10.99 & 23.24 & 10.70 & 22.79 & 9.11 \\
\hline Duration (s) & 32 & 0.59 & 0.39 & 0.53 & 0.26 & 0.85 & 0.35 & 0.69 & 0.26 \\
\hline Kinematic stiffness $\left(s^{-1}\right)$ & 32 & 7.37 & 1.95 & 7.49 & 3.96 & 6.01 & 3.02 & 5.45 & 2.16 \\
\hline \multicolumn{10}{|l|}{ Tongue dorsum } \\
\hline Amplitude (mm) & 32 & 11.34 & 3.04 & 11.37 & 3.11 & 4.14 & 1.76 & 4.17 & 1.81 \\
\hline Peak velocity $(\mathrm{mm} / \mathrm{s})$ & 32 & 65.18 & 26.14 & 45.68 & 13.81 & 21.31 & 9.86 & 22.60 & 8.79 \\
\hline Duration (s) & 32 & 0.91 & 0.39 & 0.67 & 0.18 & 0.61 & 0.30 & 0.52 & 0.23 \\
\hline Kinematic stiffness $\left(s^{-1}\right)$ & 32 & 5.97 & 1.41 & 5.24 & 1.71 & 7.22 & 2.60 & 6.63 & 1.41 \\
\hline
\end{tabular}

a Trial-sets; each trial-set comprises a series of eight repeated swallows. 
Table 5. Descriptive statistics for within-trial-set variation in movement amplitude, peak velocity, duration, and kinematic stiffness during discrete water swallows.

\begin{tabular}{|c|c|c|c|c|c|c|c|c|c|}
\hline & \multirow[b]{2}{*}{$N^{a}$} & \multicolumn{2}{|c|}{ Up } & \multicolumn{2}{|c|}{ Down } & \multicolumn{2}{|c|}{ Forward } & \multicolumn{2}{|c|}{ Backward } \\
\hline & & $M$ & $S D$ & M & $S D$ & $M$ & $S D$ & $M$ & $S D$ \\
\hline \multicolumn{10}{|l|}{ Tongue blade } \\
\hline Amplitude (mm) & 32 & 1.47 & 0.91 & 1.49 & 0.66 & 1.66 & 0.71 & 1.57 & 0.52 \\
\hline Peak velocity $(\mathrm{mm} / \mathrm{s})$ & 32 & 8.58 & 6.69 & 9.40 & 5.59 & 8.05 & 3.68 & 8.89 & 3.78 \\
\hline Duration (s) & 32 & 0.28 & 0.12 & 0.32 & 0.12 & 0.38 & 0.19 & 0.29 & 0.15 \\
\hline Kinematic stiffness $\left(s^{-1}\right)$ & 32 & 2.53 & 1.00 & 3.88 & 4.46 & 2.97 & 1.43 & 2.57 & 0.95 \\
\hline \multicolumn{10}{|l|}{ Tongue body } \\
\hline Amplitude (mm) & 32 & 3.21 & 2.01 & 3.36 & 1.97 & 1.96 & 0.92 & 2.04 & 0.66 \\
\hline Peak velocity $(\mathrm{mm} / \mathrm{s})$ & 32 & 19.48 & 9.89 & 11.96 & 5.87 & 8.08 & 3.28 & 7.99 & 4.04 \\
\hline Duration (s) & 32 & 0.23 & 0.12 & 0.26 & 0.13 & 0.47 & 0.20 & 0.35 & 0.14 \\
\hline Kinematic stiffness $\left(s^{-1}\right)$ & 32 & 2.47 & 1.44 & 5.17 & 5.14 & 3.39 & 4.36 & 2.09 & 1.32 \\
\hline \multicolumn{10}{|l|}{ Tongue dorsum } \\
\hline Amplitude (mm) & 32 & 3.73 & 2.28 & 4.56 & 2.98 & 2.43 & 0.99 & 2.18 & 0.77 \\
\hline Peak velocity $(\mathrm{mm} / \mathrm{s})$ & 32 & 21.27 & 10.57 & 14.75 & 7.38 & 10.61 & 3.75 & 9.67 & 3.44 \\
\hline Duration (s) & 32 & 0.38 & 0.24 & 0.34 & 0.14 & 0.39 & 0.23 & 0.28 & 0.14 \\
\hline Kinematic stiffness $\left(\mathrm{s}^{-1}\right)$ & 32 & 1.59 & 0.93 & 3.36 & 4.29 & 4.08 & 3.02 & 2.68 & 0.80 \\
\hline
\end{tabular}

a Trial-sets; each trial-set comprises a series of eight repeated swallows.

T3 X-ray microbeam pellet movement on $2 \mathrm{cc}$ and $10 \mathrm{cc}$ water swallows. It is encouraging to note the strong correspondence between the amplitude data in this study and those of Martin (1991) and Tasko et al., particularly given the fact that the transducer coil locations used in the present study differ somewhat from those in previous $\mathrm{X}$ ray microbeam investigations. This concurs with similar findings reported by Byrd et al. (1999) in a comparison of EMMA and X-ray microbeam speech data; we conclude that slight variation in coil placement does not pose significant challenges to the interpretation of the data.

However, the mean peak velocity values obtained in the current analysis appear substantially lower than those reported by Martin (1991) and Tasko et al. (2002), whereas movement durations appear substantially longer. Comparison of peak velocity and movement duration data across studies must be performed with caution, given that procedural differences exist in the methods used. In Martin's analysis, movements were measured along a principal-component axis rather than in $X$ and $Y$ directions orthogonal to the occlusal plane; consequently, orientation-specific aspects of movement control were not explored. In our procedures, a lower frequency cut-off for data filtering was used $(6 \mathrm{~Hz})$ than the $10 \mathrm{~Hz}$ cut-off used in the X-ray microbeam database analyses. Both Martin and Tasko et al. segmented their data using zerocrossings in the velocity and acceleration time histories, whereas in the current analysis we used an automated peak-picking procedure based on the cSTI. Martin's data were segmented into functional legs (stages) of lingual movement, which do not correspond directly to the direction-specific movement segments analyzed in the present report. Similarly, Tasko et al.'s data refer to events in a $1000 \mathrm{~ms}$ window, centered on a dominant peak velocity spike in the tongue dorsum (Tasko et al., 2002), whereas the present data capture all events for each swallow-toswallow interval in the trial-set. Finally, in the current analysis, trial-set mean values for the kinematic variables are computed as the average of all movement cycles across the eight swallows in each trial-set; this differs from previous studies in which these measures were made using single minima and maxima for isolated discrete swallow tokens. These differences are considered likely to contribute to the observed differences in results for peak velocity and movement duration measures.

\section{Discussion}

The data reported in this manuscript illustrate that EMMA is both a useful and feasible method for pointparameterization of tongue movements during swallowing, and permits detailed exploration of the influences of experimental task manipulations on swallowing behaviors. Although not illustrated in this report, EMMA may also be used to trace and measure mandibular movements during swallowing, and therefore it lends itself to future studies of coordinative relationships between articulators in the swallowing synergy (e.g., the tongue and jaw), such as those recently described by Green and Wang (2003). Future analyses will also explore the effects of 
manipulating swallowing rate and bolus consistency on tongue behaviors. Our experimental protocol therefore offers rich potential as a method for studying systematic variations in oral motor behaviors that potentially reflect underlying motor control mechanisms for swallowing.

A number of procedural and technical issues must be carefully considered with respect to conducting and analyzing EMMA studies of oral articulatory movement during swallowing. Our experience suggests that participants do not have difficulties tolerating the procedure, even when data collection sessions exceed $1 \mathrm{hr}$ in duration. However, EMMA studies of swallowing face a particular challenge, in comparison to studies of speech production, in that the transducer coils can become dislodged or break under the shearing forces of bolus propulsion during swallowing. It may therefore be advisable to plan a priori to complete multiple data collection sessions (spaced at least 1 week apart) with each participant, and subsequently to discard data from sessions in which a high rate of coil breakage or displacement is encountered.

EMMA provides a restricted 2-D midsagittal representation of tongue movements and can only sample discrete positions (in this case, three) on the tongue. Previous authors have concluded that tongue movements for bolus accommodation and propulsion occur primarily along the midsagittal groove of the tongue (Hamlet, Stone, \& Shawker, 1988; Kahrilas, Lin, Logemann, Ergun, \& Facchini, 1993). The present data suggest that the EMMA system is able to capture the main degrees of freedom for oral tongue movements (blade, body, and dorsum) during swallowing (see Figure 2). EMMA shares with other tongue movement tracing techniques the inability to relate specific movement features to bolus location.

In summary, the present report demonstrates the feasibility of using EMMA to study oral articulatory behaviors during swallowing, through an analysis of discrete water swallows from 8 normal participants. To our knowledge, this is the first published report in which EMMA has been used to investigate patterns of tongue movements during swallowing. This technique offers advantages over traditional clinical radiographic procedures, in the ability to collect larger samples of data from individual participants without biohazard and to quantify tongue movements with greater spatial and temporal accuracy.

\section{References}

Beck, T. J., \& Gayler, B. W. (1991). Radiation in videorecorded fluoroscopy. In B. Jones \& M. W. Donner (Eds.), Normal and abnormal swallowing: Imaging in diagnosis and therapy (pp. 1-6). New York: Springer-Verlag.

Byrd, D., Browman, C. P., Goldstein, L., \& Honorof, D. (1999). Magnetometer and X-ray microbeam comparison. In J. J. Ohala, Y. Hasegawa, M. Ohala, D. Granville, \&
A. C. Bailey (Eds.), Proceedings of the 14th International Congress of Phonetic Sciences (pp. 627-630). New York: American Institute of Physics.

Chi-Fishman, G., Stone, M., \& McCall, G. N. (1998). Lingual action in normal sequential swallowing. Journal of Speech, Language, and Hearing Research, 41, 771-785.

Cichero, J. A., \& Murdoch, B. E. (2002). Acoustic signature of the normal swallow: Characterization by age, gender, and bolus volume. Annals of Otology, Rhinology, and Laryngology, 111, 623-632.

Engelke, W., Schönle, P. W., Kring, R. A., \& Richter, R. C. (1989). Zur Untersuchung orofazialer Bewegungsfunktionen mit der elektromagnetischen Artikulographie [Electromagnetic articulography (EMA) studies on orofacial movement functions]. Deutsche Zahnarztliche Zeitschrift, 44, 618-622.

Green, J. R., \& Wang, Y.-T. (2003). Tongue surface movement patterns during speech and swallowing. Journal of the Acoustical Society of America, 113, 2820-2833.

Hamlet, S. L., Nelson, R. J., \& Patterson, R. L. (1990). Interpreting the sounds of swallowing: Fluid flow through the cricopharyngeus. Annals of Otology, Rhinology, and Laryngology, 99, 749-752.

Hamlet, S. L., Stone, M., \& Shawker, T. H. (1988). Posterior tongue grooving in deglutition and speech: Preliminary observations. Dysphagia, 3, 65-68.

Hasegawa-Johnson, M. (1998). Electromagnetic exposure safety of the Carstens articulograph AG100. Journal of the Acoustical Society of America, 104, 2529-2532.

Kahrilas, P. J., Lin, S., Logemann, J. A., Ergun, G. A., \& Facchini, F. (1993). Deglutitive tongue action: Volume accommodation and bolus propulsion. Gastroenterology, 104, 152-162.

Martin, R. E. (1991). A comparison of lingual movement in swallowing and speech production. Unpublished doctoral dissertation, the University of Wisconsin, Madison.

The Mathworks, Inc. (2001). MATLAB (Version 6.0.0.42a, Release 12) (Computer software). Natick, MA: Author.

Munhall, K. G., Ostry, D. J., \& Parush, A. (1985). Characteristics of velocity profiles of speech movements. Journal of Experimental Psychology: Human Perception and Performance, 11, 457-474.

Nicosia, M. A., Hind, J. A., Roecker, E. B., Carnes, M., Doyle, J., Dengel, G. A., et al. (2000). Age effects on the temporal evolution of isometric and swallowing pressure. Journals of Gerontology Series A: Biological Sciences and Medical Sciences, 55, M634-M640.

Perkell, J. S., \& Zandipour, M. (2002). Economy of effort in different speaking conditions. II. Kinematic performance spaces for cyclical and speech movements. Journal of the Acoustical Society of America, 112, 16421651.

Smith, A., Goffman, L., Zelaznik, H. N., Ying, G., \& McGillem, C. (1995). Spatiotemporal stability and patterning of speech movement sequences. Experimental Brain Research, 104, 493-501.

Steele, C. M., \& Van Lieshout, P. H. H. M. (2002). Kinematics of swallowing measured using electromagnetic midsagittal articulography [Abstract]. Dysphagia, 17,176 . 
Steele, C. M., \& Van Lieshout, P. H. H. M. (2003). Withinsubject variability in swallowing kinematics [Abstract]. Dysphagia, 18, 156.

Tasko, S. M., Kent, R. D., \& Westbury, J. R. (2002). Variability in tongue movement kinematics during normal liquid swallowing. Dysphagia, 17, 126-138.

Van Lieshout, P. H. H. M., Alfonso, P. J., Hulstijn, W., \& Peters, H. F. M. (1994). Electromagnetic midsagittal articulography (EMMA). In F. J. Maarse, A. E. Akkerman, A. N. Brand, L. J. M. Mulder, \& M. J. Van der Stelt (Eds.), Applications, methods and instrumentation (pp. 62-76). Lisse, The Netherlands: Swets \& Zeitlinger B.V.

Van Lieshout, P. H. H. M., \& Moussa, W. (2000). The assessment of speech motor behaviors using electromagnetic articulography. The Phonetician, 81, 9-22.

Van Lieshout, P. H. H. M., Rutjens, C. A. W., \& Spauwen, P. H. M. (2002). The dynamics of interlip coupling in speakers with a repaired unilateral cleft lip history.
Journal of Speech, Language, and Hearing Research, 45, 5-19.

Westbury, J. (1991). The significance and measurement of head position during speech production experiments using the X-ray microbeam system. Journal of the Acoustical Society of America, 89(4, part 1), 1782-1791.

Westbury, J. R. (1994). On coordinate systems and the representation of articulatory movements. Journal of the Acoustical Society of America, 95, 2271-2273.

Received May 21, 2003

Accepted September 20, 2003

DOI: $10.1044 / 1092-4388(2004 / 027)$

Contact author: Catriona M. Steele, $\mathrm{PhD}$, Graduate Department of Speech Pathology, University of Toronto, 500 University Avenue, Mail Room 160, Toronto, Ontario, Canada M5G 1V7. E-mail: catriona.steele@utoronto.ca 